\title{
A statistical model for expected cycle time of SP-AS/RS: an application of Monte Carlo simulation
}

\begin{abstract}
The elimination of international trade barriers, lower tariffs, and shifting centers of global manufacturing and consumption leads to new dynamics in intermodal shipping. Advanced technologies, and in particular, automated storage/retrieval systems (AS/RSs) for designing high-capacity automated container terminals, have been recently proposed as possible candidates for improving the terminal's efficiency and meeting the challenges of the future in marine transportation. In this article, the authors present an analytical statistical model for computing expected cycle time of split-platform AS/RS (SP-AS/RS), in order to reduce average handling time of this system. The accuracy of the proposed statistical model is validated by Monte Carlo simulation. The results show that the analytical model is reliable for the design and analysis of the SP-AS/RS.
\end{abstract}

Keyword: Expected cycle time; Automatic retrieval and storage system; Monte Carlo simulation; SP-AS/RS 